\title{
COMPARISON OF BIRKHOFF TYPE QUADRATURE FORMULAE
}

\author{
BORISLAV BOJANOV AND GENO NIKOLOV
}

\begin{abstract}
The classical approach to the theory of quadrature formulae is based on the concept of algebraic degree of precision (ADP). A quadrature formula $Q_{1}$ is considered to be "better" than $Q_{2}$ if $\operatorname{ADP}\left(Q_{1}\right)>\operatorname{ADP}\left(Q_{2}\right)$. However, there are many quadratures that use the same number of evaluations of the integrand and have the same ADP. Then, how should one compare such formulae? We show in this paper that the error of the quadrature depends monotonically on the type of data used. Roughly speaking, the lower the order of the derivatives used, the smaller is the error.

As a consequence of the main result we demonstrate the existence of Birkhoff quadrature formulae of double precision.
\end{abstract}

\section{INTRODUCTION}

Let $E=\left(e_{i j}\right)_{i=0, j=0}^{m+1} N_{0}$ be a given incidence matrix, i.e., a matrix containing only 0 and 1 entries. Denote by $|E|$ the number of 1's in $E$. Without loss of generality we assume in this paper that $E$ is normal, which means that $|E|$ equals the number $N$ of columns in $E$.

Any maximal sequence of 1-entries $e_{i j}=\cdots=e_{i, j+l-1}=1$ in $E$ is called a block. The block is even (odd) if its length, i.e., the number $l$, is even (odd).

The first 1-entry of the block defines its "level". Precisely, the level of the block $e_{i j}=\cdots=e_{i, j+l-1}=1$ is $j$.

We study here quadrature formulae of the form

$$
I(f):=\int_{a}^{b} f(t) d t \approx \sum_{e_{i j}=1} a_{i j} f^{(j)}\left(x_{i}\right)=: S(f)
$$

with nodes $\mathbf{x}=\left(x_{0}, \ldots, x_{m+1}\right), a=x_{0}<x_{1}<\cdots<x_{m}<x_{m+1}=b$, and real coefficients $\left\{a_{i j}\right\}$. G. D. Birkhoff gave a kernel representation of the error of (1) in his famous paper [2].

Recall that the algebraic degree of precision (ADP) of (1) is the maximal integer $n$ such that (1) is exact for all $f \in \pi_{n}\left(\pi_{n}\right.$ denotes the set of all algebraic polynomials of degree $\leq n)$.

Received April 21, 1988; revised January 16, 1989.

1980 Mathematics Subject Classification (1985 Revision). Primary 65D30; Secondary 41A55. 
Suppose that the matrix $E$ satisfies the Pólya condition

$$
\sum_{i=0}^{m+1} \sum_{j=0}^{k} e_{i j} \geq k+1, \quad k=0, \ldots, N-1 .
$$

Then, it is not difficult to verify that

$$
\operatorname{ADP}(1) \leq \sigma(E)-1,
$$

where $\sigma(E):=|E|+$ the number of the odd blocks in the interior rows of $E$.

Indeed, add one additional 1-entry to each odd interior block to obtain a new matrix $\bar{E}=\left\{\bar{e}_{i j}\right\}$. Clearly, $\bar{E}$ has only even interior blocks, $|\bar{E}|=\sigma(E)$, and $\bar{E}$ satisfies the Pólya condition. Then, by the well-known Atkinson-Sharma theorem [1], there is a polynomial $P(t)=t^{\sigma(E)}+\cdots$ such that $P^{(j)}\left(x_{i}\right)=0$ if $\bar{e}_{i j}=1$. Since $P(t)$ has a constant sign on $[a, b], \int_{a}^{b} P(t) d t \neq 0$. Thus the rule (1) is not exact for $f=P$ and therefore $\operatorname{ADP}(1) \leq \sigma(E)-1$.

The quadrature formulae defined by an incidence matrix $E$ with odd interior blocks and which have ADP equal to $\sigma(E)-1$ are called Gaussian quadrature formulae.

We assume henceforth that the matrix $E$ has only even blocks in the interior rows. Then $E=\bar{E}$, and hence $\sigma(E)=|E|$. Note that $E$ is poised, hence, by the Atkinson-Sharma theorem [1], we can construct an interpolatory quadrature formula (1) based on $E$. By construction, $\operatorname{ADP}(1)=|E|-1$. Thus, in view of (2), for any Pólya matrix $E$ with even interior blocks, the corresponding interpolatory quadrature formula (1) has maximal ADP. One cannot distinguish between these quadratures if only their ADP and the number of the evaluations are taken into account. We shall use in this paper the remainder of the quadratures (1) to compare formulas of prescribed type.

With every pair $(\mathbf{x}, E)$ we associate the polynomial

$$
\Omega(t)=\Omega(\mathbf{x}, E ; t)=t^{N}+\cdots, \quad N:=|E|,
$$

such that

$$
\Omega^{(j)}\left(x_{i}\right)=0 \text { if } e_{i j}=1 .
$$

Note that $\Omega(t)$ does not change sign on $[a, b]$, since its zeros are of even multiplicities.

Let $P(f,(\mathbf{x}, E) ; t)$ be the polynomial of degree $N-1$ that interpolates $f$ at $(\mathbf{x}, E)$. Suppose that $f$ is $N$ times continuously differentiable. Then, as in the classical Hermitian case, one can easily show (see [2 or 11, Theorem 7.5]) that for each $t \in[a, b]$ there is a $\xi \in[a, b]$ such that

$$
f(t)-P(f,(\mathbf{x}, E) ; t)=\frac{f^{(N)}(\xi)}{N !} \Omega(\mathbf{x}, E ; t) .
$$

Integrating both sides of this equality, we get the estimation

$$
|I(f)-S(f)| \leq M\left|\int_{a}^{b} \Omega(\mathbf{x}, E ; t) d t\right|
$$


with $M:=\max _{a \leq t \leq b}\left|f^{(N)}(t)\right| / N$ !. Evidently, the quantity

$$
R(\mathbf{x}, E):=\left|\int_{a}^{b} \Omega(\mathbf{x}, E ; t) d t\right|
$$

is the major term in the estimate of the error of (1).

The relation between the Gaussian quadrature formulae and the extremal problem $\inf _{\mathbf{x}} R(\mathbf{x}, E)$ was discovered by Jacobi [7] and exploited later by many authors.

This paper is a step towards proving the following:

Conjecture. The quantity $\inf \left\{R(\mathbf{x}, E): a \leq x_{i} \leq b, i=1, \ldots, m\right\}$ decreases when the level of the blocks in $E$ falls.

We give here a proof in the special case only when $E$ satisfies the condition

(i) Each interior row of $E$ contains only one block, and it has length 2 .

We prove also that the infimum is attained for some points $x_{1}^{*}, \ldots, x_{m}^{*}$ in $(a, b)$ which are located at the nodes of a Gaussian quadrature formula. This is a natural generalization of the famous result obtained by Gauss.

\section{AN AUXILIARY RESULT}

We give in this section an auxiliary result which is of some independent interest for Birkhoff interpolation.

Denote by $\mathbf{e}_{i}=\left(e_{i 0}, \ldots, e_{i, N-1}\right)$ the rows of $E=\left(e_{i j}\right)_{i=0, j=0}^{m+1} N-1$. Given the points $\mathbf{x}=\left(x_{0}, \ldots, x_{m+1}\right), a=x_{0} \leq \cdots \leq x_{m+1}=b$, we say that the function $f$ vanishes at $(\mathbf{x}, E)$, and write

$$
f_{\mid(\mathbf{x}, E)}=0
$$

if

$$
f^{(j)}\left(\tau_{i}\right)=0 \text { for } s_{i j}=1,
$$

where $\left\{\tau_{i}\right\}$ are the distinct points in the sequence $\left\{x_{0}, \ldots, x_{m+1}\right\}$ and $\left\{s_{i j}\right\}$ are the entries of the coalesced matrix $c(E)$. Recall that $c(E)$ is defined on the basis of $E$ and $\mathbf{x}$ by the so-called method of coalescence (see [10 or 11]). In other words, if $x_{p-1}<x_{p}=\cdots=x_{q}<x_{q+1}$, then the rows $\mathbf{e}_{p}, \ldots, \mathbf{e}_{q}$ of $E$ are presented in $c(E)$ as one new row $\left(s_{0}, \ldots, s_{N-1}\right)$, formed by the procedure:

1) Add $\mathbf{e}_{p}+\cdots+\mathbf{e}_{q}$ to obtain $\left(s_{0}, \ldots, s_{N-1}\right)$.

2) If $s_{i}>1$, set $s_{i}:=s_{i}-1, s_{i+1}:=s_{i+1}+1(i=0, \ldots, N-2)$.

3) Repeat 2) if necessary.

The determinant

$$
D=D(\mathbf{x}, E)=\operatorname{det}\left[\begin{array}{c}
\left\{1, t, \ldots, t^{N-1}\right\} \\
(\mathbf{x}, E)
\end{array}\right]
$$

of the system $\left.\left\{\sum_{l=0}^{N-1} \alpha_{l} t^{l}\right\}\right|_{(\mathbf{x}, E)}=0$ plays an essential role in our study. 
If the matrix $E$ satisfies the Pólya condition and if it has only even blocks, then it follows from the Atkinson-Sharma theorem [1] that

$$
D(\mathbf{x}, E) \neq 0
$$

for every $\mathbf{x}$. The next lemma asserts even more. Before formulating it, we introduce some notations which will be used throughout this paper.

Let $\alpha$ be an arbitrary fixed integer, $1 \leq \alpha \leq m$. Delete $\mathbf{e}_{\alpha}$ from $E$ and denote the resulting matrix by $E_{\alpha}$.

For any $\xi \in(a, b)$, denote by $\mathbf{x}(\xi)$ the set of points $\left(x_{0}, \ldots, x_{\alpha-1}, x_{\alpha+1}\right.$, $\left.\ldots, x_{m+1}, \xi\right)$, ordered in nondecreasing order. Set

$$
E(\xi):=E_{\alpha} \cup \mathbf{e}_{\alpha},
$$

where the row $\mathbf{e}_{\alpha}$ is inserted in the position corresponding to the position of $\xi$ in $\mathbf{x}(\xi)$.

Lemma 1. Suppose that the incidence matrix $E$ satisfies the Pólya condition and contains only even blocks in its interior rows. Then the determinant

$$
D\left(\mathbf{x},\left(\mathbf{e}_{0}, \mathbf{e}_{i_{1}}, \ldots, \mathbf{e}_{i_{m}}, \mathbf{e}_{m+1}\right)\right)
$$

has a constant sign for every rearrangement $\left(\mathbf{e}_{i_{1}}, \ldots, \mathbf{e}_{i_{m}}\right)$ of the rows $\left(\mathbf{e}_{1}, \ldots\right.$, $\left.\mathbf{e}_{m}\right)$ and each $\mathbf{x}=\left(x_{0}, \ldots, x_{m+1}\right), a=x_{0}<x_{1} \leq \cdots \leq x_{m}<x_{m+1}=b$.

Proof. It is clear that the lemma will be proved if we show that, given any $\alpha \in\{1, \ldots, m\}$, the determinant

$$
D(\xi):=D(\mathbf{x}(\xi), E(\xi))
$$

has a constant sign for each $\xi$ in $(a, b)$. In order to see this, observe that the function $D(\xi)$ has possible break points only at $\xi=x_{i}, i=1, \ldots, \alpha-1$, $\alpha+1, \ldots, m$. Therefore, the only thing we need to show is that

$$
\operatorname{sign} D\left(x_{i}-\delta\right)=\operatorname{sign} D\left(x_{i}\right)=\operatorname{sign} D\left(x_{i}+\delta\right)
$$

for sufficiently small $\delta$.

Since any even block can be considered as a coalescence of several blocks of length 2, we may restrict ourselves in this proof to the case when $\mathbf{e}_{\alpha}$ contains exactly one block, and it has length 2 and level $k-1$.

Consider the function

$$
D_{0}(\xi):=\left\{\begin{array}{l}
D(\xi) \text { for } \xi \neq x_{1}, \ldots, x_{m}, \\
\lim _{\eta \rightarrow x_{i}} D(\eta) \text { for } \xi=x_{i}, i=1, \ldots, m .
\end{array}\right.
$$

This is a polynomial function. Then, by Taylor's formula,

$$
D_{0}(\xi)=\sum_{j} D_{0}^{(j)}\left(x_{i}\right)\left(\xi-x_{i}\right)^{j} / j !
$$

Let $e_{i, k-1+n_{1}}$ and $e_{i, k+n_{2}}$ be the first two zeros in the sequence $e_{i, k-1}$, $e_{i, k}, \ldots, e_{i, N-1}$. It is easy to see that $D_{0}^{(j)}\left(x_{i}\right)=0$ for $j=0, \ldots, n-1$, where $n:=n_{1}+n_{2}$. In addition,

$$
D_{0}^{(n)}\left(x_{i}\right)=M D\left(x_{i}\right)
$$


with some positive integer $M$. Therefore,

$$
D_{0}(\xi)=M D\left(x_{i}\right)\left(\xi-x_{i}\right)^{n} / n !+O\left(\left|\xi-x_{i}\right|^{n+1}\right) \text {. }
$$

Since $\mathbf{e}_{i}$ contains only even blocks of 1 's, the number $n_{2}-n_{1}$, and consequently $n$, is even. Then

$$
\operatorname{sign} D_{0}\left(x_{i} \pm \delta\right)=\operatorname{sign} D\left(x_{i}\right)
$$

for sufficiently small $\delta>0$. The lemma is proved.

\section{THE MAIN COMPARISON RESULT}

Most of the propositions we prove in this paper deal with matrices $E$ that have the following structure:

(ii) For a fixed $\alpha \in\{1, \ldots, m\}$, the row $\mathbf{e}_{\alpha}$ contains only one block, and it has length 2. All interior rows of $E_{\alpha}$ contain only even blocks, and $E_{\alpha}$ satisfies the Pólya condition.

Set $\mathbf{x}_{\alpha}:=\mathbf{x} \backslash x_{\alpha}$. Then, by the Atkinson-Sharma theorem [1], the Birkhoff interpolation problem, based on $\left(\mathbf{x}_{\alpha}, E_{\alpha}\right)$, is poised.

Denote by $\rho(t)=t^{N-2}+\cdots$ the polynomial which vanishes at $\left(\mathbf{x}_{\alpha}, E_{\alpha}\right)$. Define the polynomial $\varphi(t)=t^{N-1}+\cdots$ by the condition

$$
\left\{\begin{array}{l}
\int_{a}^{b} \varphi(t) d t=0, \\
\varphi_{\mid\left(\mathbf{x}_{i r}, E_{a}\right)}=0 .
\end{array}\right.
$$

It is not difficult to show that the linear system (4) with respect to the coefficients of $\varphi$ has always only one solution. Indeed, assume the contrary. Then there exists a nonzero polynomial $\varphi_{0} \in \pi_{N-2}$ which satisfies (4). But the condition $\int_{a}^{b} \varphi_{0}(t) d t=0$ implies that $\varphi_{0}(t)$ has a zero of odd multiplicity which is, evidently, not specified in $\left(\mathbf{x}_{\alpha}, E_{\alpha}\right)$. Then, by the Atkinson-Sharma theorem, $\varphi_{0}(t) \equiv 0$, a contradiction.

It follows from Rolle's theorem that $\varphi^{(k-1)}(t)$ has zeros which are not specified in $\left(\mathbf{x}_{\alpha}, E_{\alpha}\right)$. Moreover, all zeros of $\varphi^{(k-1)}(t)$ that are not postulated in (4) are produced by Rolle's theorem from $\left(\mathbf{x}_{\alpha}, E_{\alpha}\right)$ and $\zeta$, where $\zeta$ is that zero of $\varphi$ whose existence is guaranteed by the first equation in (4). Let us call them Rolle's zeros. They lie at distinct points $t_{1}<\cdots<t_{r}$ in $(a, b)$, and their number $r$ can be determined exactly on the basis of the columns $0,1, \ldots, k-2$ of $E_{\alpha}$.

Denote by $e_{i, \mu_{i}-1}$ and $e_{i, \lambda_{i}}$, respectively, the first and the second 0-entry in the sequence $e_{i, k-1}, e_{i k}, \ldots, e_{i, N-1}$. Define

$$
J_{0}:=\left\{i: 0<i<m+1, \varphi^{(k-1)}\left(x_{i}\right)=0, \mu_{i}=\lambda_{i}=k\right\} .
$$

The set $\left\{t_{i}\right\}_{1}^{r}$ consists of all simple zeros $\xi_{1}, \ldots, \xi_{q}$ of $\varphi^{(k-1)}(t)$ that are not specified in $\left(\mathbf{x}_{\alpha}, E_{\alpha}\right)$, and all points $\left\{x_{i}: i \in J\right\}$ where

$$
J:=\left\{i: i \neq \alpha, 0<i<m+1, \varphi^{\left(\mu_{i}-1\right)}\left(x_{i}\right)=0\right\} \backslash J_{0} .
$$


Clearly,

$$
\begin{cases}\varphi^{(k)}\left(\xi_{i}\right) \neq 0, & i=1, \ldots, q, \\ \varphi^{\left(\lambda_{i}\right)}\left(x_{i}\right) \neq 0 & \text { if } i \in J .\end{cases}
$$

Otherwise, it would follow that $\varphi \equiv 0$.

Let $\Omega_{\xi}(t)=t^{N}+\cdots$ be the polynomial which vanishes at $(\mathbf{x}(\xi), E(\xi))$. Set, for simplicity,

$$
R_{\xi}:=R(\mathbf{x}(\xi), E(\xi))=\left|\int_{a}^{b} \Omega_{\xi}(t) d t\right| .
$$

Theorem 1. Let the normal incidence matrix $E=\left(e_{i j}\right)_{i=0, j=0}^{m+1 ~ N-1}$ and the number $\alpha$ satisfy (ii). Given arbitrary nodes $a=x_{0}<x_{1}<\cdots<x_{\alpha-1}<x_{\alpha+1}<\cdots<$ $x_{m}<x_{m+1}=b$, let

$$
\min \left\{R_{\xi}: a \leq \xi \leq b\right\}=R_{\xi^{*}}
$$

Then

$$
a<\xi^{*}<b
$$

and

$$
\varphi^{(k-1)}\left(\xi^{*}\right)=0
$$

where $k-1$ is the level of the block in $\mathbf{e}_{\alpha}$.

Moreover, if $\xi^{*}=x_{i}$ for some $i \in\{1, \ldots, m\}$, then

$$
\varphi^{\left(\mu_{i}-1\right)}\left(\xi^{*}\right)=0
$$

where $e_{i, \mu_{i}-1}$ is the first 0 in the sequence $e_{i, k-1}, e_{i k}, \ldots, e_{i, N-1}$.

The proof of this theorem goes through several lemmas.

Lemma 2. The polynomial $\rho$ satisfies

$$
\begin{aligned}
& \rho^{(k-1)}\left(\xi_{i}\right) \neq 0 \quad \text { for } i=1, \ldots, q, \\
& \rho^{\left(\mu_{i}-1\right)}\left(x_{i}\right) \neq 0 \quad \text { for } i \in J .
\end{aligned}
$$

Proof. Let $\xi \neq x_{i}, i \in J$. Summing the first $N-1$ (and $N$ ) columns of $D(\xi)$, multiplied by the coefficients of $\rho$ (and $\varphi$, respectively), we get

$$
D(\xi)=\operatorname{det}\left(\begin{array}{cc}
\rho^{(k-1)}(\xi) & \varphi^{(k-1)}(\xi) \\
\rho^{(k)}(\xi) & \varphi^{(k)}(\xi)
\end{array}\right) \cdot A,
$$

where

$$
A:=\operatorname{det}\left(\begin{array}{c}
\left\{1, t, \ldots, t^{N-3}\right\} \\
\left(\mathbf{x}_{\alpha}, E_{\alpha}\right)
\end{array}\right) .
$$

Evidently, by the Atkinson-Sharma theorem, $A \neq 0$, and $A$ does not depend on $\xi$. Moreover, in view of Lemma $1, D(\xi)$ has a constant sign for each $\xi$ in $(a, b)$. Let

$$
\varepsilon:=\operatorname{sign}[A \cdot D(\xi)]
$$


If $\xi=\xi_{i}$, we get

$$
D\left(\xi_{i}\right)=A \cdot \rho^{(k-1)}\left(\xi_{i}\right) \cdot \varphi^{(k)}\left(\xi_{i}\right)
$$

Hence,

$$
\operatorname{sign} \rho^{(k-1)}\left(\xi_{i}\right)=\varepsilon \cdot \operatorname{sign} \varphi^{(k)}\left(\xi_{i}\right), \quad i=1, \ldots, q .
$$

Similarly, in the case $i \in J$, we get

and therefore,

$$
D\left(x_{i}\right)=\operatorname{det}\left(\begin{array}{cc}
\rho^{\left(\mu_{i}-1\right)}\left(x_{i}\right) & \varphi^{\left(\mu_{i}-1\right)}\left(x_{i}\right) \\
\rho^{\left(\lambda_{i}\right)}\left(x_{i}\right) & \varphi^{\left(\lambda_{i}\right)}\left(x_{i}\right)
\end{array}\right) \cdot A
$$

$$
\operatorname{sign} \rho^{\left(\mu_{i}-1\right)}\left(x_{i}\right)=\varepsilon \cdot \operatorname{sign} \varphi^{\left(\lambda_{i}\right)}\left(x_{i}\right) \quad \text { for } i \in J .
$$

The assertion of the lemma follows from (9) and (10), on the basis of (5).

The following lemma shows that the zeros of $\varphi^{(k-1)}$ and $\rho^{(k-1)}$ interlace in a certain sense.

Lemma 3. The derivative $\rho^{(k-1)}$ has at least one zero in $\left(t_{j}, t_{j+1}\right)(j=1, \ldots$, $r-1)$ which is not specified by $\left(\mathbf{x}_{\alpha}, E_{\alpha}\right)$.

Proof. Let us first illustrate the proof of this claim in the special case when $t_{j}=\xi_{i}, t_{j+1}=\xi_{i+1}$, and $\varphi^{(k-1)}(t)$ has zeros $\left\{x_{l}\right\}$ of total multiplicity $n$ in $\left(t_{j}, t_{j+1}\right)$.

Note that all these zeros $\left\{x_{l}\right\}$ are specified by $\left(\mathbf{x}_{\alpha}, E_{\alpha}\right)$. Thus, $\rho^{(k-1)}(t)$ vanishes also at $\left\{x_{l}\right\}$.

Since $\varphi^{(k-1)}$ has $n+2$ zeros in $\left[t_{j}, t_{j+1}\right], \varphi^{(k)}$ will have, by Rolle's theorem, $n+1+n_{0}$ zeros in $\left(t_{j}, t_{j+1}\right)=\left(\xi_{i}, \xi_{i+1}\right)$. Here, $n_{0}$ is an even number, in view of assumption (ii). Thus,

$$
\operatorname{sign} \varphi^{(k)}\left(\xi_{i}\right) \cdot \operatorname{sign} \varphi^{(k)}\left(\xi_{i+1}\right)=(-1)^{n+n_{0}+1}=(-1)^{n+1}
$$

Now the relation $(9)$ implies

$$
\operatorname{sign} \rho^{(k-1)}\left(\xi_{i}\right) \cdot \operatorname{sign} \rho^{(k-1)}\left(\xi_{i+1}\right)=(-1)^{n+1} .
$$

But all zeros $\left\{x_{l}\right\}$ of $\rho^{(k-1)}$ in $\left(t_{j}, t_{j+1}\right)$, which are prescribed by $\left(\mathbf{x}_{\alpha}, E_{\alpha}\right)$, have total multiplicity $n$. Then the relation (11) shows that $\rho^{(k-1)}$ must have at least one more zero in $\left(t_{j}, t_{j+1}\right)$.

Consider another case:

$$
t_{j}=\xi_{i_{1}}, \quad t_{j+1}=x_{i} \quad \text { for some } i \in J
$$

and $\varphi^{(k-1)}$ has zeros $\left\{x_{l}\right\}$ of total multiplicity $n$ in $\left(t_{j}, t_{j+1}\right)$.

Since $\varphi^{(k-1)}$ has $1+n+\left(\lambda_{i}-k+1\right)$ zeros in $\left[t_{j}, t_{j+1}\right], \varphi^{(k)}$ will have, by Rolle's theorem, $n+\lambda_{i}-k+1+n_{0}$ zeros in $\left(t_{j}, t_{j+1}\right]$, with some even number $n_{0}$. Then,

$$
\operatorname{sign} \varphi^{(k)}\left(\xi_{i_{1}}\right) \cdot \operatorname{sign} \varphi^{\left(\lambda_{i}\right)}\left(x_{i}\right)=(-1)^{n+\lambda_{i}-k+1} .
$$


Taking into account (9) and (10), we get

$$
\operatorname{sign} \rho^{(k-1)}\left(\xi_{i_{1}}\right) \cdot \operatorname{sign} \rho^{\left(\mu_{i}-1\right)}\left(x_{i}\right)=(-1)^{n+\lambda_{i}-k+1}=(-1)^{n+\mu_{i}-k+1},
$$

since $\lambda_{i}-\mu_{i}$ is even.

Now observe that $\rho^{(k-1)}\left(x_{i}\right) \neq 0$ if $\mu_{i}=k$ and $\rho^{(k-1)}\left(x_{i}\right)=\cdots=\rho^{\left(\mu_{i}-2\right)}\left(x_{i}\right)$ $=0$ if $\mu_{i}>k$. Recall, in addition, that all zeros $\left\{x_{l}\right\}$ of $\rho^{(k-1)}$ in $\left(t_{j}, t_{j+1}\right)$, which are specified in $\left(\mathbf{x}_{\alpha}, E_{\alpha}\right)$, have total multiplicity $n$. Then, in view of (12), $\rho^{(k-1)}$ must have at least one more zero in $\left(t_{j}, t_{j+1}\right)$.

The verification of the lemma is similar in the remaining cases and is omitted here.

Proof of Theorem 1. Suppose that $\xi^{*}$ is an extremal point, i.e., that

$$
R_{\xi^{*}} \leq R_{\xi} \quad \text { for each } \xi \in[a, b]
$$

Set, for simplicity, $R:=R_{\xi^{*}}, \Omega:=\Omega_{\xi^{*}}$.

Consider the interpolation problem

$$
\left.\left\{c_{1} \varphi(t)+c_{2} \rho(t)+\sum_{i=3}^{N} c_{i} t^{N-i}\right\}\right|_{(\mathbf{x}, E)}=\left.\left(\Omega-\Omega_{\xi}\right)\right|_{(\mathbf{x}, E)}
$$

with respect to $\left\{c_{i}\right\}_{1}^{N}$. Since $\varphi, \rho$, and $\left\{t^{i}\right\}_{0}^{N-3}$ are linearly independent, there exists a unique solution $\left\{c_{i}(\xi)\right\}$ to this problem. Then,

$$
\left.\sum_{i=3}^{N} c_{i}(\xi) t^{N-i}\right|_{\left(\mathbf{x}_{i}, E_{a}\right)}=0
$$

Thus, $c_{i}(\xi)=0$ for $i \geq 3$. Therefore,

$$
\Omega(t)=\Omega_{\xi}(t)+c_{1}(\xi) \varphi(t)+c_{2}(\xi) \rho(t) .
$$

Note that $\operatorname{sign} \rho(t)=\operatorname{sign} \Omega_{\xi}(t)$ and $\int_{a}^{b} \varphi(t) d t=0$. We assume for convenience that $\Omega(t) \geq 0$ on $[a, b]$. Then the assumption (13) leads to the inequality

$$
c_{2}(\xi) \leq 0 \text { for each } \xi \in[a, b] .
$$

Next, we get from (14)

$$
\begin{gathered}
\Omega^{(k-1)}\left(\xi_{i}\right)=c_{2}\left(\xi_{i}\right) \cdot \rho^{(k-1)}\left(\xi_{i}\right), \quad i=1, \ldots, q, \\
\Omega^{\left(\mu_{i}-1\right)}\left(x_{i}\right)=c_{2}\left(x_{i}\right) \cdot \rho^{\left(\mu_{i}-1\right)}\left(x_{i}\right), \quad i \in J .
\end{gathered}
$$

Therefore,

$$
\begin{cases}\Omega^{(k-1)}\left(\xi_{i}\right) \cdot \rho^{(k-1)}\left(\xi_{i}\right) \leq 0 & \text { for } i=1, \ldots, q, \\ \Omega^{\left(\mu_{i}-1\right)}\left(x_{i}\right) \cdot \rho^{\left(\mu_{i}-1\right)}\left(x_{i}\right) \leq 0 & \text { for } i \in J\end{cases}
$$

Add also the fact that

$$
\operatorname{sign} \Omega^{(k-1)}(a+\delta)=\operatorname{sign} \rho^{(k-1)}(a+\delta)
$$


for sufficiently small $\delta>0$. To establish (16), we note that $e_{0, N-2}=e_{0, N-1}=$ 0 , since $E_{\alpha}$ is a Pólya matrix. This yields $\operatorname{sign} \Omega^{(N-2)}(a)=\operatorname{sign} \rho^{(N-2)}(a)$. Then the equality (16) follows immediately from Lemma 2.2 of Jetter [8], which asserts that $\Omega^{(l)}(a)=0$ if and only if $e_{0 l}=1$ and, equivalently, $\Omega^{(l)}(a+\delta)$. $\Omega^{(l+1)}(a+\delta)>0$ for small $\delta>0$.

The next argument presents the main idea of the proof of the theorem.

Suppose that $R<R_{\xi}$ for each $\xi \in\left\{t_{1}, \ldots, t_{r}\right\}$. This leads to strict inequalities in (15). Then the same arguments as in the proof of Lemma 3 show that $\Omega^{(k-1)}(t)$ has at least one Rolle's zero in $\left(t_{i}, t_{i+1}\right)$ for $i=1, \ldots, r-1$. Now observe that the number of Rolle's zeros of $\Omega^{(k-1)}(t)$ and $\rho^{(k-1)}(t)$ is determined by $\mathbf{x}_{\alpha}$ and columns $0, \ldots, k-2$ of $E_{\alpha}$. Since the same part of $E_{\alpha}$, together with the point $\zeta$, implies $r$ Rolle's zeros of $\varphi^{(k-1)}(t)$, it follows that $\Omega^{(k-1)}(t)$ and $\rho^{(k-1)}(t)$ have $r-1$ Rolle's zeros in $(a, b)$. Thus, all Rolle's zeros of $\Omega^{(k-1)}$ and $\rho^{(k-1)}$ lie in $\left(t_{1}, t_{r}\right)$. On the other hand, the inequalities (15) and (16) imply that $\Omega^{(k-1)}$ or $\rho^{(k-1)}$ must have at least one Rolle's zero in $\left(a, t_{1}\right)$, a contradiction.

So, we proved that

$$
\min \left\{R_{\xi}: \xi \in[a, b]\right\}=\min \left\{R_{\xi}: \xi \in\left\{t_{1}, \ldots, t_{r}\right\}\right\} .
$$

However, the theorem was formulated in a slightly stronger form. It remains to show that the minimum of $R_{\xi}$ on $[a, b]$ cannot be attained at a point $\xi$ distinct from the Rolle's zeros $t_{1}, \ldots, t_{r}$ of $\varphi^{(k-1)}$. In order to do this, we shall apply the same idea just demonstrated above.

Suppose that $\xi^{*} \notin\left\{t_{1}, \ldots, t_{r}\right\}$. Then some of the relations (15) turn into equalities. But this is not a serious difficulty. A careful study of the behavior of $\Omega^{(k-1)}(t)$ and $\rho^{(k-1)}(t)$ near the points $t_{1}, \ldots, t_{r}$, based on the inequalities (15), shows that for sufficiently small $\delta>0$, there exist points $t_{i}^{0} \in\left(t_{i}-\delta, t_{i}+\right.$ $\delta), i=1, \ldots, r$, such that

$$
\Omega^{(k-1)}\left(t_{i}^{0}\right) \cdot \rho^{(k-1)}\left(t_{i}^{0}\right)<0 .
$$

The observation (17) is obvious if $c_{2}\left(t_{i}\right)<0$. Otherwise, we have to take into account the fact that $\Omega^{(k-1)}(t)$ and $\rho^{(k-1)}(t)$ have a zero at $t_{i}$ of different parity, i.e., of even, odd multiplicity, or vice-versa. (This is the point where we use the assumption that $\xi^{*} \neq t_{1}, \ldots, t_{r}$.)

We proceed as before. It follows from Lemma 3 and (17) that $\Omega^{(k-1)}$ (and $\left.\rho^{(k-1)}\right)$ has at least one Rolle's zero in $\left(t_{i}^{0}, t_{i+1}^{0}\right)$. Then (16) leads to a contradiction. Thus $\xi^{*} \in\left\{t_{1}, \ldots, t_{r}\right\}$. This implies (6). The theorem is proved.

Now we are prepared to prove our first comparison result.

Theorem 2. Let the normal incidence matrix $E=\left(e_{i j}\right)_{i=0, j=0}^{m+1 ~} N-1$ and the number $\alpha$ satisfy (ii). Push down the block in the row $\mathbf{e}_{\alpha}$ of $E$ one position (i.e., decrease the level $k-1$ of the block by 1$)$ and denote the resulting matrix by $\widehat{E}$. Given 
arbitrary nodes $a=x_{0}<x_{1}<\cdots<x_{\alpha-1}<x_{\alpha+1}<\cdots<x_{m}<x_{m+1}=b$, the following inequality is true:

$$
\min _{a \leq \xi \leq b} R(\mathbf{x}(\xi), \widehat{E}(\xi)) \leq \min _{a \leq \xi \leq b} R(\mathbf{x}(\xi), E(\xi)) .
$$

Proof. It is known (see [6]) that the Birkhoff interpolating polynomial $P(f,(\mathbf{x}, E) ; t)$ depends continuously on $\mathbf{x}$. Then $\int_{a}^{b} \Omega_{\xi}(t) d t$ is a continuous function of $\xi$, and therefore $R_{\xi}$ attains its minimal value at some point $\xi^{*}$ in $[a, b]$. According to the Characterization Theorem $1, \xi^{*}$ satisfies (6), (7), and (8). Denote now by $b_{j}(\xi)$ the coefficient of $f^{(j)}(\xi)$ in the interpolatory quadrature formula (1), based on $(\mathbf{x}(\xi), E(\xi))$. We claim that

$$
\begin{cases}b_{k}\left(\xi^{*}\right)=0 & \text { if } \xi^{*} \in\left\{\xi_{1}, \ldots, \xi_{q}\right\}, \\ b_{\lambda_{i}}\left(\xi^{*}\right)=0 & \text { if } \xi^{*}=x_{i} \text { for some } i \in J .\end{cases}
$$

Indeed, by Theorem 1 , the extremal point $\xi^{*}$ coincides with some Rolle's zero of the corresponding polynomial $\varphi^{(k-1)}(t)$. Then in both cases the coefficient $b\left(\xi^{*}\right)$ from (18) equals const $\cdot \int_{a}^{b} \varphi(t) d t$. But the integral is equal to zero, in view of (4). The proof of claim (18) is completed.

Let $\Omega$ and $\widehat{\Omega}$ be the polynomials of degree $N$ with leading coefficient 1 , which vanish at $\left(\mathbf{x}\left(\xi^{*}\right), E\left(\xi^{*}\right)\right)$ and $\left(\mathbf{x}\left(\xi^{*}\right), \widehat{E}\left(\xi^{*}\right)\right)$, respectively. Since $f(t):=$ $\Omega(t)-\widehat{\Omega}(t)$ is a polynomial of degree $\leq N-1$ and $\operatorname{ADP}(1)=N-1$, we get

$$
\int_{a}^{b} f(t) d t=b_{k}\left(\xi^{*}\right) \cdot f^{(k)}\left(\xi^{*}\right)=0
$$

in the case $\xi^{*} \in\left\{\xi_{1}, \ldots, \xi_{q}\right\}$. Thus,

$$
R_{\xi^{*}}=R\left(\mathbf{x}\left(\xi^{*}\right), \widehat{E}\left(\xi^{*}\right)\right) \geq \min _{a \leq \xi \leq b} R(\mathbf{x}(\xi), \widehat{E}(\xi)) .
$$

Similarly, one can verify (19) in the case $\xi^{*} \in\left\{x_{i}: i \in J\right\}$. The theorem is proved.

Remark 1. The next simple example shows that the error $R(\mathbf{x}, E)$ does not always decrease when we reduce the level of the blocks, keeping the nodes fixed.

Let $[a, b]:=[0,1], m=1$,

$$
E=\left[\begin{array}{lll}
1 & 0 & 0 \\
0 & 1 & 1 \\
0 & 0 & 0
\end{array}\right], \quad \widehat{E}=\left[\begin{array}{lll}
1 & 0 & 0 \\
1 & 1 & 0 \\
0 & 0 & 0
\end{array}\right], \quad \mathbf{x}=(0, \xi, 1) .
$$

Then, $\Omega(t)=t^{3}-3 \xi t^{2}+3 \xi^{2} t, \widehat{\Omega}(t)=t(t-\xi)^{2}$, and therefore

$$
\begin{aligned}
& R_{\xi}=\int_{0}^{1} \Omega(t) d t=\frac{1}{4}-\xi+\frac{3}{2} \xi^{2}, \\
& \widehat{R}_{\xi}=\int_{0}^{1} \widehat{\Omega}(t) d t=\frac{1}{4}-\frac{2}{3} \xi+\frac{1}{2} \xi^{2} .
\end{aligned}
$$

Clearly, $R_{\xi}<\widehat{R}_{\xi}$ for any fixed $\xi \in\left(0, \frac{1}{3}\right)$. 
Remark 2. The following example shows that it is not possible to prove strict inequality in the assertion of Theorem 2.

Let $[a, b]:=[0,1], m=2, \alpha=2$,

$$
\begin{gathered}
E=\left[\begin{array}{llllll}
1 & 1 & 0 & 0 & 0 & 0 \\
0 & 1 & 1 & 0 & 0 & 0 \\
0 & 0 & 1 & 1 & 0 & 0 \\
0 & 0 & 0 & 0 & 0 & 0
\end{array}\right], \\
\widehat{E}=\left[\begin{array}{llllll}
1 & 1 & 0 & 0 & 0 & 0 \\
0 & 1 & 1 & 0 & 0 & 0 \\
0 & 1 & 1 & 0 & 0 & 0 \\
0 & 0 & 0 & 0 & 0 & 0
\end{array}\right], \quad \mathbf{x}=\left(0, x_{1}, \xi, 1\right) .
\end{gathered}
$$

Observe that the functions $\varphi$ and $\rho$, introduced at the beginning of this section, are the same for $E$ and $\widehat{E}$. Clearly, $\varphi^{\prime}$ has a unique Rolle's zero $\eta, 0<\eta<1$, produced by $x_{0}$ and $\zeta$. Moreover, $\eta$ depends continuously on $x_{1}$ in $[0,1]$, and therefore there exists a situation when $\eta \equiv x_{1}$. We shall assume here that $x_{1}$ is chosen in that way. Now, by Theorem $1, \min R(\mathbf{x}(\xi), \widehat{E}(\xi))$ is achieved only at $\xi^{*}=x_{1}$. Let

$$
\int_{a}^{b} f(t) d t \approx a_{0} f(0)+a_{1} f^{\prime}(0)+b_{1} f^{\prime}\left(x_{1}\right)+b_{2} f^{\prime \prime}\left(x_{1}\right)+b_{3} f^{\prime \prime \prime}\left(x_{1}\right)
$$

be the interpolatory quadrature formula based on $\left(\mathbf{x}\left(\xi^{*}\right), E\left(\xi^{*}\right)\right) . \quad\left(b_{4}=0\right.$, according to Theorem 1.) Since (20) is exact for $\pi_{5}$, we get

$$
\int_{0}^{1} \rho(t) d t=b_{3} \cdot \rho^{\prime \prime \prime}\left(x_{1}\right)>0 \text {. }
$$

It is easy to verify that $\rho^{\prime \prime \prime}\left(x_{1}\right)>0$. Therefore,

$$
b_{3}>0 \text {. }
$$

Note that $\varphi^{\prime \prime \prime}$ has exactly two Rolle's zeros $\xi_{1}$ and $\xi_{2}, 0<\xi_{1}<x_{1}, \xi_{2}=x_{1}$. Thus, by Theorem $1, \min R(\mathbf{x}(\xi), E(\xi))$ can be achieved at $\xi_{1}$ or $\xi_{2}$ only. Set

$$
\Omega_{i}(t):=\Omega\left(\mathbf{x}\left(\xi_{i}\right), E\left(\xi_{i}\right) ; t\right), \quad i=1,2 .
$$

Now, applying (20) to $\Omega_{2}(t)-\Omega_{1}(t)$, we get

$$
\int_{0}^{1}\left[\Omega_{2}(t)-\Omega_{1}(t)\right] d t=-b_{3} \cdot \Omega_{1}^{\prime \prime \prime}\left(x_{1}\right) .
$$

Since $\Omega_{1}^{\prime \prime \prime}\left(x_{1}\right)>0,(21)$ implies

$$
\int_{0}^{1} \Omega_{2}(t) d t<\int_{0}^{1} \Omega_{1}(t) d t
$$

Therefore,

$$
\min _{0 \leq \xi \leq 1} R(\mathbf{x}(\xi), E(\xi))=R\left(\mathbf{x}\left(\xi_{2}\right), E\left(\xi_{2}\right)\right)=\min _{0 \leq \xi \leq 1} R(\mathbf{x}(\xi), \widehat{E}(\xi)) .
$$




\section{COMPARISON WITH RESPECT TO THE ENDPOINT TERMS}

The previous theorem treats interior blocks only. Next we shall prove a similar result about the monotone dependence of the error $R(\mathbf{x}, E)$ on the level of the 1-entries in the first and last rows of $E$.

Our proof is based on the following extension of the classical Budan-Fourier theorem.

Denote, as usual, by $S^{-}\left(f_{1}, \ldots, f_{n}\right)$ the number of the strong sign changes in the sequence of numbers $f_{1}, \ldots, f_{n}$. Let $S^{+}\left(f_{1}, \ldots, f_{n}\right)$ denote the maximal number of sign changes in $f_{1}, \ldots, f_{n}$, where the zeros are interpreted as -1 or +1 . Let Int $E$ be the interior of $E$ (i.e., the matrix obtained from $E$ by deletion of the first and last row).

Lemma 4. Suppose that the incidence matrix $E=\left(e_{i j}\right)_{i=0, j=0}^{m+1} N-1$ satisfies the Pólya condition and all non-Hermitian blocks of Int $E$ are even.

Then

$$
|\operatorname{Int} E| \leq S^{-}\left(f(a), \ldots, f^{(N)}(a)\right)-S^{+}\left(f(b), \ldots, f^{(N)}(b)\right)
$$

for each polynomial $f$ of exact degree $N$ which vanishes at $(\mathbf{x}, E)$ for some points $a=x_{0}<\cdots<x_{m}<x_{m+1}=b$.

The lemma is an immediate consequence of Theorem 2.5 in [11]. The proof is given there.

Suppose that the incidence vectors $\mathbf{e}=\left(e_{1}, \ldots, e_{N-1}\right)$ and $\widehat{\mathbf{e}}=\left(\widehat{e}_{1}, \ldots\right.$, $\hat{e}_{N-1}$ ) have the same number of 1-entries. Let

$$
\begin{aligned}
& e_{i}=1 \quad \text { if and only if } i=\lambda_{1}, \ldots, \lambda_{n}, \\
& \hat{e}_{i}=1 \quad \text { if and only if } i=\hat{\lambda}_{1}, \ldots, \hat{\lambda}_{n} .
\end{aligned}
$$

We write here, for the sake of simplicity, $\mathbf{e} \leq \widehat{\mathbf{e}}$ if $\lambda_{i} \leq \hat{\lambda}_{i}$ for $i=1, \ldots, n$.

Theorem 3. Let the incidence matrices $E=\left(e_{i j}\right)_{i=0, j=0}^{m+1} N-1$ and $\widehat{E}=\left(\widehat{e}_{i j}\right)_{i=0, j=0}^{m+1} N-1$ have only even blocks of 1 's in the interior rows and satisfy the Polya condition. Suppose that $\mathbf{e}_{i}=\widehat{\mathbf{e}}_{i}$ for $i=1, \ldots, m$ and $\widehat{\mathbf{e}}_{0} \leq \mathbf{e}_{0}, \widehat{\mathbf{e}}_{m+1} \leq \mathbf{e}_{m+1}$.

Then

$$
R(\mathbf{x}, \widehat{E}) \leq R(\mathbf{x}, E)
$$

for each $\mathbf{x}=\left(x_{0}, \ldots, x_{m+1}\right), a=x_{0}<x_{1}<\cdots<x_{m+1}=b$.

Moreover, equality holds if and only if $E \equiv \widehat{E}$.

Proof. We use here an idea employed in [4, Theorem 3.5].

Suppose that

$$
\begin{gathered}
e_{0, j}=1 \quad \text { if and only if } j=\lambda_{1}, \ldots, \lambda_{n}, \\
e_{m+1, j}=1 \quad \text { if and only if } i=\mu_{1}, \ldots, \mu_{n_{1}} .
\end{gathered}
$$

Similarly, the 1-entries of $\widehat{\mathbf{e}}_{0}$ and $\widehat{\mathbf{e}}_{m+1}$ are prescribed by $\left\{\hat{\lambda}_{i}\right\}_{1}^{n}$ and $\{\hat{\mu}\}_{1}^{n_{1}}$. 
Evidently, the assertion will follow by pairwise comparisons and symmetry, if we prove that

$$
R(\mathbf{x}, \widehat{E})<R(\mathbf{x}, E)
$$

in the case

$$
\begin{array}{ll}
\mu_{i}=\widehat{\mu}_{i}, & i=1, \ldots, n_{1}, \\
\lambda_{i}=\hat{\lambda}_{i} & \text { for all } i \neq k, \\
\hat{\lambda}_{k}=\lambda_{k}-1 & \text { where } \lambda_{k}-1 \notin\left\{\lambda_{1}, \ldots, \lambda_{n}\right\} .
\end{array}
$$

In order to do this, we study the polynomials $\Omega$ and $\widehat{\Omega}$ defined by $(\mathbf{x}, E)$ and $(\mathbf{x}, \widehat{E})$, respectively. Without loss of generality, we may assume that they are nonnegative on $[a, b]$. Suppose that $\widehat{\Omega}(\tau)>\Omega(\tau)>0$ for some $\tau \in(a, b)$. Consider the polynomial

$$
g(t):=\Omega(t)-\beta \cdot \widehat{\Omega}(t),
$$

where $\beta:=\Omega(\tau) / \widehat{\Omega}(\tau)$. Clearly, $0<\beta<1$. Then $g(t)$ is of degree $N$ and has a leading coefficient of the same sign as that of $\Omega$. In addition,

$$
\left\{\begin{array}{l}
g(\tau)=0, \\
g^{(j)}\left(x_{i}\right)=0 \text { if } e_{i j}=1, i=1, \ldots, m,
\end{array}\right.
$$

and all these zeros are prescribed by an incidence matrix $E_{1}$ which satisfies the requirements of Lemma 4 . Note that $\left|\operatorname{Int} E_{1}\right|=|\operatorname{Int} E|+1=N-n-n_{1}+1$. Therefore, by Lemma 4,

$$
N-n-n_{1}+1 \leq S^{-}\left(g(a), \ldots, g^{(N)}(a)\right)-S^{+}\left(g(b), \ldots, g^{(N)}(b)\right) .
$$

It then follows from the obvious estimations

$$
\begin{aligned}
& S^{-}\left(g(a), \ldots, g^{(N)}(a)\right) \leq N-n+1, \\
& S^{+}\left(g(b), \ldots, g^{(N)}(b)\right) \geq n_{1}
\end{aligned}
$$

that (23) is actually an equality. Thus,

$$
S^{-}\left(g(a), \ldots, g^{(N)}(a)\right)=N-n+1 .
$$

On the other hand, since

$$
\begin{gathered}
\operatorname{sign} g^{(N)}(a)=\operatorname{sign} \Omega^{(N)}(a), \\
\operatorname{sign} g^{\left(\lambda_{k}\right)}(a)=-\operatorname{sign} \widehat{\Omega}^{\left(\lambda_{k}\right)}(a)=\operatorname{sign} \Omega^{(\lambda)}(a),
\end{gathered}
$$

where $e_{0 \lambda}$ is the first 0 -entry in the sequence $e_{0, \lambda_{k}+1}, \ldots, e_{0, N-1}$, we conclude (see [4] for details) that

$$
S^{-}\left(g(a), \ldots, g^{(N)}(a)\right) \leq S^{-}\left(\Omega(a), \ldots, \Omega^{(N-1)}(a)\right)=N-n,
$$

a contradiction. Thus, $\widehat{\Omega}(t) \leq \Omega(t)$ for each $t \in[a, b]$. This implies (22), since $\Omega \not \equiv \widehat{\Omega}$. The proof is completed. 


\section{EXISTENCE OF BIRKHOFF QUADRATURES OF DOUBLE PRECISION}

The fundamental question of existence of Gaussian quadrature formulae of preassigned non-Hermitian type was studied first by Micchelli and Rivlin [12] (see also Dyn [5]).

We say that the quadrature formula $Q$ has double precision if it uses $N$ evaluations and $\operatorname{ADP}(Q)=2 N-1$. Clearly, any such formula is defined by a matrix $E$ that has blocks of length 1 only. For example, the Gauss quadrature formula has double precision. Jetter [8] proved recently the existence of pyramidal Birkhoff quadratures of double precision. We shall derive here an existence theorem treating the general case. The major ingredient of our proof is the characterization result of Theorem 1 , combined with the technique of splitting nodes, described in the next propositions.

Lemma 5. Let the incidence matrix $E=\left(e_{i j}\right)_{i=0, j=0}^{m+1} N-1$ satisfy the Pólya condition. Suppose that all interior blocks of $E$ are even. Let $\left\{a_{i j}\right\}$ be the coefficients of the interpolatory quadrature formula (1) based on $(\mathbf{x}, E)$ for some fixed nodes $\mathbf{x}=\left(x_{0}, \ldots, x_{m+1}\right), a=x_{0}<x_{1}<\cdots<x_{m+1}=b$. Let $\left(e_{i j}, e_{i, j+1}, \ldots, e_{i n}\right), n-j \geq 3$, be an arbitrary interior block of $E$. Suppose that

1) $a_{i, k}=0$ for some $k \in\{j+3, \ldots, n\}$;

2) $\boldsymbol{\Omega}^{(n+1)}\left(\mathbf{x}, E ; x_{i}\right) \neq 0$;

3) $n-k$ is an even number.

Then for each $j \leq r \leq k-2$ there exists a pair $\left(\mathbf{x}_{h}, E_{i}\right)$ such that

$$
\left|\int_{a}^{b} \Omega\left(\mathbf{x}_{h}, E_{i} ; t\right) d t\right|<\left|\int_{a}^{b} \Omega(\mathbf{x}, E ; t) d t\right| .
$$

The pair $\left(\mathbf{x}_{h}, E_{i}\right)$ is of the form

or

$$
\left\{\begin{array}{l}
\mathbf{x}_{h}=\left(x_{0}, \ldots, x_{i}, x_{i}+h, x_{i+1}, \ldots, x_{m+1}\right), h>0 ; E_{i} \text { is ob- } \\
\text { tained from } E \text { by setting } e_{i, k-1}=e_{i, k}=0 \text { in } \mathbf{e}_{i} \text { and inserting a } \\
\text { new row } \mathbf{e} \text { between } \mathbf{e}_{i} \text { and } \mathbf{e}_{i+1} \text { which has } 1 \text { 's only in positions } \\
r \text { and } r+1 ;
\end{array}\right.
$$

$$
\text { (B) } \quad\left\{\begin{array}{l}
\mathbf{x}_{h}=\left(x_{0}, \ldots, x_{i-1}, \tau-h, x_{i}, \tau+h, x_{i+1}, \ldots, x_{m+1}\right), h>0 ; \\
E_{i} \text { is obtained from } E \text { by setting } e_{i j}=\cdots=e_{i k}=0 \text { in } \mathbf{e}_{i} \text { and } \\
\text { inserting two new rows } \mathbf{e}_{\tau-h} \text { and } \mathbf{e}_{\tau+h} \text { corresponding to the points } \\
\tau-h \text { and } \tau+h, \text { one of which has } 1 \text { 's in positions } j, \ldots, k-2, \\
\text { and the other in } r, r+1 \text { only. }
\end{array}\right.
$$

Proof. Since the polynomials $\Omega_{h}(t):=\Omega\left(\mathbf{x}_{h}, E_{i} ; t\right), \Omega(t):=\Omega(\mathbf{x}, E ; t)$ have the same sign in $(a, b)$, we assume below for convenience that $\Omega_{h}(t) \geq 0$, $\Omega(t) \geq 0$ in $(a, b)$.

Suppose that there is no $\mathbf{x}_{h}$ of the form (A) for which (24) holds. Then

$$
\int_{a}^{b}\left[\Omega_{h}(t)-\Omega(t)\right] d t \geq 0
$$


for each sufficiently small $h>0$. Since $a_{i k}=0$ and the quadrature (1) based on $(\mathbf{x}, E)$ is interpolatory, we get

$$
\int_{a}^{b}\left[\Omega_{h}(t)-\Omega(t)\right] d t=a_{i, k-1} \cdot \Omega_{h}^{(k-1)}\left(x_{i}\right) \geq 0 .
$$

Now note that the coefficients of the polynomial $\Omega_{h}(t)$ depend continuously on $h$. This follows from the fact that $E$ is a coalescence matrix of $E_{i}$ and from a general result about the continuity of the Birkhoff interpolating polynomial (see [6]). Then, by Markov's inequality for the derivatives of algebraic polynomials,

$$
\left\|\Omega_{h}^{(n+1)}-\Omega^{(n+1)}\right\|_{C[a, b]} \rightarrow 0 \quad \text { as } h \rightarrow 0 .
$$

Therefore, in view of assumption 2), there exists $h_{0}>0$ such that $\Omega_{h}^{(n+1)}(t) \neq 0$ on $\left(x_{i}-h, x_{i}+h\right)$ for any $h \in\left[0, h_{0}\right]$. Moreover,

$$
\operatorname{sign} \Omega_{h}^{(n+1)}(t)=\operatorname{sign} \Omega^{(n+1)}\left(x_{i}\right)
$$

for $t \in\left(x_{i}-h, x_{i}+h\right)$. Using Rolle's theorem, the assumption $j \leq r \leq k-2$, and (26), we conclude that $\Omega_{h}^{(k-1)}(t)$ has exactly two zeros in $\left(x_{i}, x_{i}+h\right]$, $\Omega_{h}^{(k)}(t)$ has exactly one zero, and $\Omega_{h}^{(k+1)}(t)$ has no zero in $\left(x_{i}, x_{i}+h\right)$. Thus, $\Omega_{h}^{(k-1)}\left(x_{i}\right) \neq 0$. In addition,

$$
\operatorname{sign} \Omega_{h}^{(k+1)}(t)=\operatorname{sign} \Omega_{h}^{(k+2)}(t)=\cdots=\operatorname{sign} \Omega_{h}^{(n+1)}(t)=\operatorname{sign} \Omega^{(n+1)}\left(x_{i}\right)
$$

for $t \in\left(x_{i}, x_{i}+h\right)$. This follows from the well-known fact that $f(c+t)$. $f^{\prime}(c+t)>0$ for any small $t>0$ if the algebraic polynomial $f$ vanishes at $c$. Therefore,

$$
\operatorname{sign} \Omega_{h}^{(k-1)}\left(x_{i}\right)=\operatorname{sign} \Omega_{h}^{(k+1)}(t)=\operatorname{sign} \Omega^{(n+1)}\left(x_{i}\right)
$$

for $t \in\left(x_{i}, x_{i}+h\right)$ and hence, on the basis of (25),

$$
\operatorname{sign} a_{i, k-1}=\operatorname{sign} \Omega^{(n+1)}\left(x_{i}\right) .
$$

Observe that $a_{i, k-1} \neq 0$ if $a_{i k}=0$. (Otherwise, the quadrature (1) cannot be exact for $\pi_{N-1}$.)

In the preceding discussion, $\mathbf{x}_{h}$ was assumed to be of the form (A). Next, we assume that $\mathbf{x}_{h}$ is of the form (B) with $h$ and $\tau$ such that

$$
\Omega_{h}^{(k)}\left(x_{i}\right)=0 \text {. }
$$

Then many of the above conclusions about $\Omega_{h}$ remain true also in case (B). In particular,

$$
\operatorname{sign} \Omega_{h}^{(k+1)}(t)=\operatorname{sign} \Omega^{(n+1)}\left(x_{i}\right) \text { for } t \in\left(x_{i}, \tau+h\right),
$$

and since $n-k$ is an even number, the latter holds also for $t \in\left(\tau-h, x_{i}\right)$.

As in case (A), we conclude that $\Omega_{h}^{(k-1)}(t)$ has exactly two zeros $\alpha<\beta$ in $(\tau-h, \tau+h)$, and then

$$
\operatorname{sign} \Omega_{h}^{(k-1)}(t)=-\operatorname{sign} \Omega^{(n+1)}\left(x_{i}\right)
$$


for $t \in(\alpha, \beta)$. It follows from (28) and (26) that

$$
\max _{\alpha \leq t \leq \beta}\left|\Omega_{h}^{(k-1)}(t)\right|=\left|\Omega_{h}^{(k-1)}\left(x_{i}\right)\right|=: \rho .
$$

Then,

$$
\left|\boldsymbol{\Omega}^{(\lambda)}\left(x_{i}\right)\right| \leq \rho \cdot(2 h)^{k-1-\lambda}, \quad \lambda=j, \ldots, k-2 .
$$

(This is a standard technique. The details can be found, for example, in [3].)

Applying the interpolatory quadrature formula (1) based on $(\mathbf{x}, E)$ and taking into account (30), we get

$$
\begin{aligned}
\int_{a}^{b}\left[\Omega_{h}(t)-\Omega(t)\right] d t & =\sum_{\lambda=j}^{n} a_{i \lambda} \cdot \Omega_{h}^{(\lambda)}\left(x_{i}\right) \\
& =a_{i, k-1} \cdot \Omega_{h}^{(k-1)}\left(x_{i}\right)+\rho \cdot O(h) \\
& =\rho \cdot\left[a_{i, k-1} \cdot \operatorname{sign} \Omega_{h}^{(k-1)}\left(x_{i}\right)+O(h)\right] .
\end{aligned}
$$

Since $\alpha<x_{i}<\beta$, (29) implies

$$
\operatorname{sign} \Omega_{h}^{(k-1)}\left(x_{i}\right)=-\operatorname{sign} \Omega^{(n+1)}\left(x_{i}\right)
$$

and hence, by (27),

$$
a_{i, k-1} \cdot \operatorname{sign} \Omega_{h}^{(k-1)}\left(x_{i}\right)=-\left|a_{i, k-1}\right| .
$$

Recall that $a_{i, k-1} \neq 0$. Thus,

$$
\int_{a}^{b}\left[\Omega_{h}(t)-\Omega(t)\right] d t<0
$$

for sufficiently small $h$. The proof is completed.

Lemma 6. Let the normal incidence matrix $E=\left(e_{i j}\right)_{i=0, j=0}^{m+1 ~} N-1$ satisfy the Pólya condition and have only even blocks in the interior rows. Suppose that for a fixed $i \in\{1, \ldots, m\}$ the row $\mathbf{e}_{i}$ contains exactly one block $\left(e_{i \lambda}, \ldots, e_{i n}\right)$. Given the nodes $\mathbf{x}=\left(x_{0}, \ldots, x_{m+1}\right), a=x_{0}<\cdots<x_{m+1}=b$, denote by $\left\{a_{k j}(\xi)\right\}$ the coefficients of the interpolatory quadrature formula based on $(\mathbf{x}(\xi), E)$, where $\xi$ is a parameter and

$$
\mathbf{x}(\xi)=\left(x_{0}, \ldots, x_{i-1}, \xi, x_{i+1}, \ldots, x_{m+1}\right) .
$$

Suppose that

$$
a_{i n}(\tau)=0 \quad \text { and } \quad \Omega^{(n+1)}(\mathbf{x}(\tau), E ; \tau)=0
$$

for some $\tau \in(a, b)$ such that

(a) $\tau=x_{i}$; or

(b) $\tau=x_{l}$ for some $l \neq i$ with the property that $\mathbf{e}_{i}$ and $\mathbf{e}_{l}$ are not in collision (i.e., $e_{i k} \cdot e_{l k}=0$ for $k=0, \ldots, N-1$ ) and $e_{l, n+1}=0$.

Then $\xi=\tau$ is not an extremal point for the integral

$$
I(\xi):=\int_{a}^{b} \Omega(\mathbf{x}(\xi), E ; t) d t .
$$


Proof. We shall show first that

$$
\left.\frac{\partial I}{\partial \xi}\right|_{\xi=\tau}=-a_{i n}(\tau) \cdot \Omega^{(n+1)}(\mathbf{x}(\tau), E ; \tau) .
$$

Indeed, set for convenience

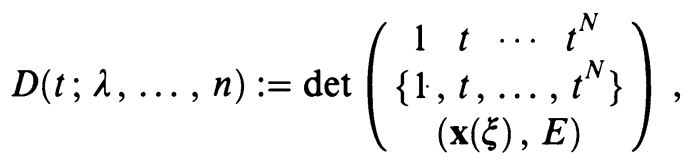

where $\lambda, \ldots, n$ indicate the order of the derivatives in the consecutive rows containing $\xi$. Similarly,

$$
D=D_{N}(\lambda, \ldots, n):=\operatorname{det}\left(\begin{array}{c}
\left\{1, t, \ldots, t^{N-1}\right\} \\
(\mathbf{x}(\xi), E)
\end{array}\right) .
$$

We have

$$
I(\xi)=\frac{\int_{a}^{b} D(t ; \lambda, \ldots, n) d t}{D_{N}(\lambda, \ldots, n)} .
$$

Suppose that $\xi$ is in a neighborhood of a point $\tau$ satisfying (a) or (b). Then

$$
\begin{aligned}
\frac{\partial I}{\partial \xi}=\frac{1}{D^{2}} \int_{a}^{b}[D(t ; \lambda, \ldots, n-1, n+1) \cdot D & -D(t ; \lambda, \ldots, n) \\
& \left.\cdot D_{N}(\lambda, \ldots, n-1, n+1)\right] d t .
\end{aligned}
$$

Since the integrand is a polynomial of degree $N-1$, we apply the interpolatory quadrature formula based on $(\mathbf{x}(\xi), E)$ and get

$$
\frac{\partial I}{\partial \xi}=\left.\frac{a_{i n}(\xi)}{D} \cdot \frac{\partial^{n}}{\partial t^{n}} D(t ; \lambda, \ldots, n-1, n+1)\right|_{t=\xi} .
$$

Observe that

$$
\left.\frac{\partial^{n}}{\partial t^{n}} D(t ; \lambda, \ldots, n-1, n+1)\right|_{t=\xi}=-\left.\frac{\partial^{n+1}}{\partial t^{n+1}} D(t ; \lambda, \ldots, n)\right|_{t=\xi} .
$$

Thus,

$$
\frac{\partial I}{\partial \xi}=-a_{i n}(\xi) \cdot \Omega^{(n+1)}(\mathbf{x}(\xi), E ; \xi)
$$

which yields $(31)$ for $\xi=\tau$.

We now consider separately two cases.

Case A. Let $\tau=x_{i}$. Note that

$$
\begin{aligned}
\Omega^{(n+1)}(\mathbf{x}(\xi), E ; \xi) & =\left.\frac{1}{D} \frac{\partial^{n+1}}{\partial t^{n+1}} D(t ; \lambda, \ldots, n)\right|_{t=\xi} \\
& =(-1)^{\mu} \frac{D_{N+1}(\lambda, \ldots, n, n+1)}{D_{N}(\lambda, \ldots, n)},
\end{aligned}
$$


where $\mu:=\left|\mathbf{e}_{0}\right|$ and $D_{N+1}$ is defined in a similar way as $D_{N}(\lambda, \ldots, n)$. Then,

$$
\begin{aligned}
\frac{\partial}{\partial \xi} \Omega^{(n+1)}(\mathbf{x}(\xi), E ; \xi) \\
\quad=(-1)^{\mu} \frac{D_{N+1}(\lambda, \ldots, n, n+2) \cdot D-D_{N+1}(\lambda, \ldots, n, n+1) \cdot D_{N}(\lambda, \ldots, n-1, n+1)}{D^{2}} .
\end{aligned}
$$

Since

$$
\begin{aligned}
& D_{N+1}(\lambda, \ldots, n, n+2) / D=(-1)^{\mu} \cdot \Omega^{(n+2)}(\mathbf{x}(\xi), E ; \xi), \\
& D_{N+1}(\lambda, \ldots, n, n+1) / D=(-1)^{\mu} \cdot \Omega^{(n+1)}(\mathbf{x}(\xi), E ; \xi),
\end{aligned}
$$

and $\Omega^{(n+1)}(\mathbf{x}(\tau), E ; \tau)=0$, we obtain

$$
\left.\frac{\partial}{\partial \xi} \Omega^{(n+1)}(\mathbf{x}(\xi), E ; \xi)\right|_{\xi=\tau}=(-1)^{\mu} \cdot \Omega^{(n+2)}(\mathbf{x}(\tau), E ; \tau) \text {. }
$$

Similarly, one can show that

$$
\left.\frac{\partial}{\partial \xi} a_{i n}(\xi)\right|_{\xi=\tau}=-a_{i, n-1}(\tau)
$$

if $a_{i n}(\tau)=0$. For this purpose, denote by $E_{i n}$ the matrix obtained from $E$ by setting $e_{i n}=0$. Let $\psi(\mathbf{x}(\xi), E ; t)$ be the polynomial of degree $N-1$ such that

$$
\left.\psi\right|_{\left(\mathbf{x}(\xi), E_{i n}\right)}=0, \quad \psi^{(n)}(\xi)=1
$$

Clearly,

$$
a_{i n}(\xi)=\int_{a}^{b} \psi(\mathbf{x}(\xi), E ; t) d t
$$

Using the determinant representation of $\psi(\mathbf{x}, E ; t)$ and straightforward calculations, we get (33). Note here that (33) is true for $\tau$ satisfying (b) as well. Another proof of the relation (33) was given in [9].

Now observe that

$$
\begin{aligned}
a_{i, n-1}(\tau) \neq 0 & \text { if } a_{i n}(\tau)=0, \\
\Omega^{(n+2)}(\mathbf{x}(\tau), E ; \tau) \neq 0 & \text { if } \Omega^{(n+1)}(\mathbf{x}, E ; \tau)=0 .
\end{aligned}
$$

Then, using (31), (32), and (33), we obtain

$$
\left.\frac{\partial I}{\partial \xi}\right|_{\xi=\tau}=0,\left.\quad \frac{\partial^{2} I}{\partial \xi^{2}}\right|_{\xi=\tau}=0,\left.\quad \frac{\partial^{3} I}{\partial \xi^{3}}\right|_{\xi=\tau} \neq 0 .
$$

The last relations show that $\xi=\tau$ is not an extremum point for $I(\xi)$ in Case A.

Case B. Let $\tau$ satisfy (b). The proof is identical with that in Case A if $e_{l, n+2}=$ 0 . Suppose that $e_{l, n+2}=1$. This means that $\mathbf{e}_{l}$ contains a block $\left(e_{l, n+2}, \ldots\right.$, $\left.e_{l q}\right)$ and, according to the assumptions of the lemma, $q-n$ is an odd number. 
The lemma will be proved in this case if we show that $\partial I / \partial \xi$ does not change its sign at $\xi=\tau=x_{l}$. It was established above that

$$
\begin{aligned}
\frac{\partial I}{\partial \xi} & =\left.\frac{a_{i n}(\xi)}{D} \cdot \frac{\partial^{n}}{\partial t^{n}} D(t ; \lambda, \ldots, n-1, n+1)\right|_{t=\xi} \\
& =(-1)^{\mu+1} a_{i n}(\xi) \cdot D_{N+1}(\lambda, \ldots, n-1, n, n+1) / D .
\end{aligned}
$$

Lemma 1 guarantees a constant sign for $D$. Further, since $a_{i n}(\tau)=0$ and $\left.\frac{\partial}{\partial \xi} a_{i n}(\xi)\right|_{\xi=\tau}=-a_{i, n-1}(\tau) \neq 0, a_{i n}(\xi)$ changes it sign at $\xi=\tau$.

Consider the function $D_{N+1}(\xi):=D_{N+1}(\lambda, \ldots, n-1, n, n+1)$ in a neighborhood of $\tau=x_{l}$. Using the Taylor expansion of $D_{N+1}(\xi)$ at $\xi=\tau$, we get

$$
D_{N+1}(\xi)=\frac{D_{N+1}^{(q-n)}(\tau)}{(q-n) !}(\xi-\tau)^{q-n}+O\left(|\xi-\tau|^{q+1-n}\right) .
$$

But

$$
D_{N+1}^{(q-n)}(\tau)=D_{N+1}(\lambda, \ldots, n-1, n, n+2, \ldots, q, q+1) \neq 0 .
$$

Indeed, suppose that $D_{N+1}(\lambda, \ldots, n-1, n, n+2, \ldots, q, q+1)=0$. Then there exist two polynomials $\rho_{1}$ and $\rho_{2}$ of the form $t^{N}+\cdots$ which vanish at $(\mathbf{x}(\tau), E)$ and, in addition, $\rho_{1}^{(n+1)}(\tau)=0, \rho_{2}^{(q)}(\tau)=0$. Then $\rho_{1}-\rho_{2} \in \pi_{N-1}$ and $\left(\rho_{1}-\rho_{2}\right)_{\mid(\mathbf{x}(\tau), E)}=0$. Hence $\rho_{1} \equiv \rho_{2}$, and consequently $\rho_{1} \equiv 0$, a contradiction.

Thus, $D_{N+1}^{(q-n)}(\tau) \neq 0$, and hence $D_{N+1}(\xi)$ changes its sign at $\xi=\tau$.

Then $\partial I / \partial \xi$ does not change its sign at $\xi=\tau$ and the proof is completed.

We are now ready to prove existence.

Theorem 4. Let $E=\left(e_{i j}\right)_{i=0, j=0}^{m+1 N-1}$ be an arbitrary normal incidence matrix which satisfies (i). Suppose that for each $\alpha \in\{1, \ldots, m\}$ the matrix $E_{\alpha}$ satisfies the Pólya condition. Let $k_{1}, \ldots, k_{m}$ be the levels of the interior blocks of $E$.

Then there exists a quadrature formula of the form

$$
\int_{a}^{b} f(x) d x \approx \sum_{i=1}^{m} a_{i} \cdot f^{\left(k_{i}\right)}\left(x_{i}^{*}\right)+\sum_{e_{0 j}=1} A_{j} \cdot f^{(j)}(a)+\sum_{e_{m+1, j}=1} B_{j} \cdot f^{(j)}(b)
$$

with $A D P=2 m-1+\left|\mathbf{e}_{0}\right|+\left|\mathbf{e}_{m+1}\right|$ and $x_{i}^{*} \in(a, b), i=1, \ldots, m$.

Proof. Set

$$
R(E):=\inf \left\{R(\mathbf{x}, E) ; a \leq x_{i} \leq b, i=1, \ldots, m\right\} .
$$

The polynomial $\Omega(\mathbf{x}, E ; t)$ depends continuously on $x_{i}$ (see [6 or 8, Lemma 2.2]). Then there exist points $\xi_{\nu_{1}}, \ldots, \xi_{\nu_{m}}$ such that $a \leq \xi_{\nu_{i}} \leq b, i=$ $1, \ldots, m$, and

$$
R(\bar{\xi}, E)=R(E), \quad \overline{\boldsymbol{\xi}}=\left(a, \xi_{\nu_{1}}, \ldots, \boldsymbol{\xi}_{\nu_{m}}, b\right) .
$$

Since $\xi_{\nu_{i}}$ is the extremal location of $x_{i}$, keeping all $\xi_{\nu_{j}}, j \neq i$, fixed, it follows from Theorem 1 that $a<\xi_{\nu_{i}}<b$ for each $i$. 
Next we shall show that $\xi_{\nu_{\lambda}} \neq \xi_{\nu_{\mu}}$ if their corresponding blocks are in collision, i.e., if $\left|k_{\lambda}-k_{\mu}\right|<2$. Assume the contrary. Then there is a block $\bar{\beta}=\left(s_{p}, \ldots, s_{n}\right)$ in the coalesced matrix $c(E)$ which is formed by the coalescence of interior rows of $E$, some of which are in collision. Moreover, there is a sequence $\bar{\gamma}=\left(s_{q}, \ldots, s_{k}\right)$ of 1's, $p \leq q<k \leq n$, such that each component (i.e., original block of length 2) of $\bar{\gamma}$ is in collision with the coalescence of the remaining components of $\bar{\gamma}$. Assume further that $\bar{\gamma}$ is the highest subsequence of $\bar{\beta}$ with this property and the block $\bar{\beta}_{i}$ of the row $\mathbf{e}_{i}$ has a highest level among all components of $\bar{\gamma}$. Then $\bar{\beta}_{i}$ will occupy the consecutive positions $\left(s_{k-1}, s_{k}\right)$ in the coalescence block $\bar{\beta}$. Since $\xi_{\nu_{i}}$ is the extremal location of $x_{i}$, it follows from Theorem 1 that $a_{i k}=0$, where $a_{\lambda \mu}$ denotes the coefficient of $f^{(\mu)}\left(\xi_{\nu_{\lambda}}\right)$ in the interpolatory quadrature defined by $(\overline{\boldsymbol{\xi}}, E)$. If $k=n$, then $\Omega^{(n+1)}\left(\bar{\xi}, E ; \xi_{\nu_{i}}\right) \neq 0$, by Lemma 6 , applied to the block $\bar{\beta}$. (We use here that the whole block $\bar{\beta}$ is in an optimal position if its components are optimally located.) In case $k<n$ we observe that the block $\left(s_{n-1}, s_{n}\right)$ (which is not in collision with other components of $\bar{\beta}$ ) corresponds to the optimal location $\xi_{\nu_{j}}=\xi_{\nu_{i}}$ of some $x_{j}$. Thus, by Theorem 1, $a_{i n}=0$. This yields, again by Lemma 6 , that $\Omega^{(n+1)}\left(\bar{\xi}, E ; \xi_{\nu_{i}}\right) \neq 0$. Now we can apply Lemma 5 to split the node $\xi_{\nu_{i}}$ and obtain a quadrature formula with a smaller error $R$, a contradiction. So, the blocks remain in their original levels $\left\{k_{i}\right\}$ after the optimization. Applying again Theorem 1, we get

$$
a_{i, k_{i}+1}=0, \quad i=1, \ldots, m,
$$

which completes the proof.

Let us point out that if $\mathbf{e}_{0}=\mathbf{e}_{m+1}=(0, \ldots, 0)$ in Theorem 4 , the resulting extremal quadrature formula has double precision.

It is a well-known fact that the nodes of the famous Gauss quadrature formula minimize the integral $\int_{a}^{b}\left(x-x_{1}\right)^{2} \cdots\left(x-x_{m}\right)^{2} d x$. Theorem 4 reveals a similar connection between the extremal problem

$$
\left|\int_{a}^{b} \Omega(\mathbf{x}, E ; t) d t\right| \rightarrow \min
$$

and a Gaussian quadrature formula of Birkhoff type defined by $E$.

Theorem 5. Let the incidence matrices $E=\left(e_{i j}\right)_{i=0, j=0}^{m+1} N-1$ and $\widehat{E}=\left(\widehat{e}_{i j}\right)_{i=0, j=0}^{m+1 ~ N-1}$ satisfy the requirements (i). Suppose that $E_{\alpha}$ and $\widehat{E}_{\alpha}$ satisfy the Polya condition for every $\alpha \in\{1, \ldots, m\}$. Let $\widehat{\mathbf{e}}_{0} \leq \mathbf{e}_{0}, \widehat{\mathbf{e}}_{m+1} \leq \mathbf{e}_{m+1}$. Suppose that

$$
\hat{k}_{i} \leq k_{i}, \quad i=1, \ldots, m,
$$

where $\left(\hat{k}_{i}\right)_{1}^{m}$ and $\left(k_{i}\right)_{1}^{m}$ are the levels of the blocks in the interior rows of $\widehat{E}$ and $E$, respectively. Then

$$
R(\widehat{E}) \leq R(E) .
$$


Moreover, equality is attained if and only if $E \equiv \widehat{E}$.

Proof. Assume first that $\widehat{E}$ and $E$ have the same boundary rows. There is nothing to prove if $k_{i}=\hat{k}_{i}$ for all $i$. Suppose that $\hat{k}_{i}<k_{i}$ for some fixed $i$, $1 \leq i \leq m$. Push down the block in the $i$ th interior row of $E$ one position and denote the resulting matrix by $\widehat{E}$. The assertion will follow by pairwise comparisons if we prove that $R(\widehat{E})<R(E)$.

Let $R(E)=R(\bar{\xi}, E)$ for $\bar{\xi}=\left(a, \xi_{\nu_{1}}, \ldots, \xi_{\nu_{m}}, b\right)$. Let $\left(s_{0}, \ldots, s_{N-1}\right)$ be the coalesced (possibly) row at the point $\xi_{\nu_{i}}$. Since there is no collision in this coalescence (see the proof of Theorem 4), $\left(s_{k_{i}}, s_{k_{i}+1}\right)$ is the block corresponding to $\xi_{\nu_{i}}$. Assume first that $s_{k_{i}-1}=0$. Applying the interpolatory quadrature based on $(\bar{\xi}, E)$, we get

$$
\int_{a}^{b}[\Omega(\overline{\boldsymbol{\xi}}, E ; t)-\widehat{\Omega}(\overline{\boldsymbol{\xi}}, E ; t)] d t=0 .
$$

Thus, $R(\overline{\boldsymbol{\xi}}, \widehat{E})=R(E)$. Evidently, $R(\widehat{E}) \leq R(\bar{\xi}, \widehat{E})$. We need to show that

$$
R(\widehat{E})<R(\bar{\xi}, \widehat{E}) .
$$

Assume that $R(\widehat{E})=R(\bar{\xi}, \widehat{E})$. This means that $\xi_{\nu_{i}}$ is an extremal point for the matrix $\widehat{E}$ as well. Since $s_{k_{i}-1}=0$, the polynomial $\varphi$, defined by (4), is the same for $E$ and $\widehat{E}$. Then it follows from Theorem 1 that $\varphi^{(k-1)}\left(\xi_{\nu_{i}}\right)=$ $\varphi^{(k-2)}\left(\xi_{\nu_{i}}\right)=0$ with $k-1=k_{i}$. But this leads to $\varphi \equiv 0$, a contradiction. Let now $s_{k_{i}-1}=1$. Then there is a collision between the block of $\xi_{\nu_{1}}$ and some other block in the coalescence of $\widehat{E}$ with respect to $\bar{\xi}$. This yields, as in the proof of Theorem 4, that $\bar{\xi}$ is not extremal for $\widehat{E}$. Thus $R(\widehat{E})<R(\bar{\xi}, \widehat{E})$.

The proof is completed in the case when the boundary rows of $E$ and $\widehat{E}$ coincide. Now the general result follows from the above reasoning and Theorem 3.

\section{ACKNOWLEDGMENT}

The authors are grateful to the referee for his valuable remarks and suggestions.

\section{BIBLIOGRAPHY}

1. K. Atkinson and A. Sharma, A partial characterization of poised Hermite-Birkhoff interpolation problems, SIAM J. Numer. Anal. 6 (1969), 230-235.

2. G. D. Birkhoff, General mean value theorem and remainder theorems with application to mechanical differentiation and quadrature, Trans. Amer. Math. Soc. 7 (1906), 107-136.

3. B. Bojanov, On the existence of optimal quadrature formulae for smooth functions, Calcolo 16 (1979), 61-70.

4. _ Comparison theorems in optimal recovery, in Optimal Algorithms (Bl. Sendov, ed.), Bulg. Akad. Nauk, Sofia, 1986, pp. 15-50. 
5. N. Dyn, On the existence of Hermite-Birkhoff quadrature formulas of Gaussian type, J. Approx. Theory 31 (1981), 22-32.

6. N. Dyn, G. G. Lorentz, and S. D. Riemenschneider, Continuity of the Birkhoff interpolation, SIAM J. Numer. Anal. 19 (1982), 507-509.

7. C. G. J. Jacobi, Ueber Gauss neue Metode, die Werthe der Integrale näherungsweise zu finden, J. Reine Angew. Math. 1 (1826), 301-308.

8. K. Jetter, A new class of Gaussian quadrature formulas based on Birkhoff type data, SIAM J. Numer. Anal. 19 (1982), 1081-1089.

9. __ Uniqueness of Gauss-Birkhoff quadrature formulas, SIAM J. Numer. Anal. 24 (1987), 147-154.

10. G. G. Lorentz and K. Zeller, Birkhoff interpolation problem: Coalescence of rows, Arch. Math. 26 (1975), 189-192.

11. G. G. Lorentz, K. Jetter, and S. D. Riemenschneider, Birkhoff interpolation, Encyclopedia of Mathematics and its Applications, vol. 19, Reading, Mass., 1983.

12. C. A. Micchelli and T. J. Rivlin, Quadrature formulae and Hermite-Birkhoff interpolation, Adv. in Math. 11 (1973), 93-112.

Department of Mathematics, University of Sofia, Boulevard A. Ivanov 5, 1126 Sofia, Bulgaria 